\title{
Similarity in the Amino Acid Sequences of Mycobacterium tuberculosis Protein Targets Involved in Binding Sites of Docking with Thiacetazone
}

\section{Mafakheri $\mathbf{M}^{1}$, Sardari $\mathbf{S}^{1 *}$}

Drug Design and Bioinformatics Unit, Medical Biotechnology Department, Biotechnology Research Center, Pasteur Institute, Iran

*Corresponding author: Sardari S, Drug Design and Bioinformatics Unit, Biotechnology Research Center, Pasteur Institute of Iran, Tehran 13164, Iran, E-mail: ssardari@hotmail.com

Received date: August 29, 2016; Accepted date: October 29, 2016; Published date: October 31, 2016

Copyright: () 2016 Sardari S et al. This is an open-access article distributed under the terms of the Creative Commons Attribution License, which permits unrestricted use, distribution, and reproduction in any medium, provided the original author and source are credited.

\begin{abstract}
Although according to WHO document, between 1990 and 2015, both TB mortality and its incidence has been fallen over $47 \%$ worldwide, the spread of multidrug-resistant strains of Mycobacterium tuberculosis reveals clearly that the efforts to find new drugs should not be stopped and the pathogenic microorganisms develop resistance. More extended knowledge about existing drugs is critical to design new and more effective medicines. In this study, we report the amino acid sequences involved in binding sites of $70 \mathrm{M}$. tuberculosis protein targets' docked with Thiacetazone (TAC), one of the extensively used antitubercular drug that is used in combination with other antitubercular agents to break multi-drug resistant TB. Categorization of protein targets was performed on the basis of the free energy of binding for the docked compounds. Comparison of the binding sites with the aim of ClustalW application indicated huge similarities in their amino acid sequences among target complexes.
\end{abstract}

Keywords Thiacetazone; Docking; Amino acid sequence; Binding site similarities; Mycobacterium; ClustalW; Arguslab

\section{Introduction}

Tuberculosis (TB) is a fatal contagious disease that can affect almost any part of the body especially the lungs. It is one of the top 10 causes of death worldwide. In 2015, 10.4 million people fell ill with TB and 1.8 million died from the disease [1]. Roughly one-third of the world's population has been infected with Mycobacterium tuberculosis, and new infections occur at a rate of one per second [2]. In 2015, the largest number of new TB cases occurred in Asia, with $61 \%$ of new cases, followed by Africa, with $26 \%$. It is noted that $87 \%$ of new TB cases occurred in the 30 high TB burden countries. Six countries accounted for $60 \%$ of the new TB cases: India, Indonesia, China, Nigeria, Pakistan, and South Africa. Global progress depends on advances in TB prevention and care in these countries [3]. Annual average incidence rate of tuberculosis in Iran is 17.9 in 100,000 patients [4]. Tuberculosis incidence is higher in Balochistan, Khorasan, Golestan, Gilan, Kurdistan, Western Azerbaijan, Khuzestan, and southern coasts of Iran [5]. Multi-drug resistant (MDR) strains [6], extensively drug-resistant (XDR) strains [7] and XXDR strains as GB Migliori et al., mentioned in Italy in 2007 [8], drug-resistant tuberculosis (TDR ) that has been identified in three countries; India, Iran and Italy and in all of them resistance to ordinary TB drugs is the common problem, make an important issue in TB treatment [9-11]. In 2015, an estimated 480,000 people worldwide developed MDR-TB, and an additional 100,000 people with rifampicin-resistant TB were also eligible for MDR-TB treatment. India, China, and the Russian Federation accounted for $45 \%$ of the 580,000 cases. It is estimated that about $9.5 \%$ of these cases were XDR-TB [12]. There are many efforts to combat with these drug resistant strains.

New drug synthesis and improve the properties of the old ones are included. Bedaquiline with a new mechanism of action (inhibits mycobacterial ATP synthetase and depletes cellular energy stores) is one of them. Unfortunately in one of the phase 2 studies, there were more deaths among patients who had bedaquiline added to an antimycobacterial drug regimen than among those who had placebo added to the same regimen. Therefore, FDA allows the approval of drug, only for serious or life-threatening conditions that provide meaningful therapeutic benefit over existing therapies [13]. For instance thiacetazone (Figure 1) that belong to World Health Organization group 5 drugs for the treatment of tuberculosis [14] despite cheapness and extreme usage has some serious side effect like Steven Johnson syndrome and cutaneous hypersensitivity reactions especially among patients with human immunodeficiency virus infection $[15,16]$. Hence many efforts are underway to synthesize its new and superior analogue with better properties $[17,18]$. Drugs and their targets are like lock and key, for making good key it is important to recognize and know the lock in advance and because of economical aspects, beginning such research with virtual screening is a better manner. For instance, Kandasamy et al., [19] looked at the pathogenesis of $\mathrm{TB}$ in order to find newer drugs they performed molecular docking studies with a library of kinase inhibitors. As a result T95 was found, which is a potent inhibitor for PknI, and Lys 41 along with Asp90, Val92 and Asp96 were identified as functionally important residues they suggested that docking studies helped in identifying ligand inhibitor specific to PknI which was confirmed by laboratory experimentation. Homology modelling, docking, pharmacophore and site directed mutagenesis analysis to identify the critical amino acid residue of PknI from M. tuberculosis [19]. 
Citation: Mafakheri M and Sardari S (2016) Similarity in the Amino Acid Sequences of Mycobacterium tuberculosis Protein Targets Involved in Binding Sites of Docking with Thiacetazone. Pharm Anal Acta 7: 509. doi:10.4172/2153-2435.1000509

Page 2 of 7

Figure 1: Thiacetazone.

In our study, a rapid and cheap method was used to study the amino acid sequences involved in the binding sites. This method leads to exploration of huge similarities that was not considered before.

\section{Methods}

At first the medical literature was retrospectively reviewed and well known M. tuberculosis protein target was chosen (Table 1) then they were downloaded in pdb format from protein Data Bank [20]. They were docked via Arguslab software version 4.0.1. Mark Thompson and Planaria Software LLC [21] to obtain free energy of binding measures between the thiacetazone and them. They were categorized on the basis of free energy of binding and then all amino acids involving in binding sites were recognized, briefly after docking all amino acids existed in the amino acid folder were selected, then at the binding site, it was chosen "hide" and then "show" option and after deselecting them it was chosen the "delete" option. Arguslab software deleted all amino acids except those involved in binding site. All binding site's amino acids' sequences belong to $70 \mathrm{M}$. tuberculosis target involved in docking with thiacetazone were compared via ClustalW application then it was performed a categorization on the basis of similar sequences that usually belong to same classes(Table 1) [22].

\begin{tabular}{|c|c|c|c|c|c|}
\hline No & Category & Name & Classification & Free Energy of Binding (kcal/mol) & Sequence of amino acids in Binding Site \\
\hline 1 & \multirow{15}{*}{ A } & $1 \mathrm{GSI}$ & Transferase & -9.55 & RRNDFFPSYYY \\
\hline 2 & & $1 \mathrm{MRN}$ & Transferase & -9.35 & ARRRNDDDEEEHLFFPSYYYY \\
\hline 3 & & $1 G 3 U$ & Transferase & -9.28 & RRNDFFPSYYY \\
\hline 4 & & $1 \mathrm{~W} 2 \mathrm{G}$ & Transferase & -8.97 & RRNFFPSYYY \\
\hline 5 & & $1 \mathrm{~N} 5 \mathrm{~J}$ & Transferase & -8.96 & RRNDEFFPSYYY \\
\hline 6 & & $1 \mathrm{MRS}$ & Transferase & -8.94 & RRNDLFPSYYY \\
\hline 7 & & $1 \mathrm{~N} 5 \mathrm{I}$ & Transferase & -8.78 & RRNDDLFPSYYY \\
\hline 8 & & $1 \mathrm{~W} 2 \mathrm{H}$ & Transferase & -8.59 & RRNDLFFPSYY \\
\hline 9 & & $3 F N F$ & Oxidoreductase & -8.91 & AADDGGGIIIIIILKMFFPSSTV \\
\hline 10 & & 2PR2 & Oxidoreductase & -8.69 & ADDGGGIIIIILKMMFFPSSTWYV \\
\hline 11 & & 2IEB & Oxidoreductase & -8.6 & AADDGGGIIIIILKMMMMFFPSTWYV \\
\hline 12 & & $1 Z I D$ & Oxidoreductase & -8.58 & ADDGGGIIIIILKMMMFFFPSSTWYV \\
\hline 13 & & 3FNH & Oxidoreductase & -8.47 & AADDGGGIIIIIILLKMMFFFPSSTV \\
\hline 14 & & 3FNG & Oxidoreductase & -8.45 & AADDGGGIIIIIILLKMFFPSSYV \\
\hline 15 & & $2 \mathrm{IDZ}$ & Oxidoreductase & -8.41 & ADDGGGIIIIIILKMMMFFPSSTWYV \\
\hline 16 & \multirow{9}{*}{ B } & 2IED & Oxidoreductase & -8.34 & ADDGGGIIIIILKMMFFPSSTWTVV \\
\hline 17 & & $1 \mathrm{P} 44$ & Oxidoreductase & -8.32 & ADDGGGIIIIIILKMMFFPSSTV \\
\hline 18 & & 2B35 & Oxidoreductase & -8.29 & ADDGGGIIIIILKMMFFPSSTV \\
\hline 19 & & $2 \mathrm{~B} 36$ & Oxidoreductase & -8.19 & ADDGGIIIIIILKMMMFFPSSTV \\
\hline 20 & & 2NSD & Oxidoreductase & -8.15 & ADDGGGIIIIIILKMMFFPSSTV \\
\hline 21 & & $2 \mathrm{AQI}$ & Oxidoreductase & -8.12 & ADDGGGIIIIIILKMMFFPSSTV \\
\hline 22 & & $2 \mathrm{H} 7 \mathrm{~N}$ & Oxidoreductase & -8.09 & ADDGGGIIIIIILKMMFFPSSTV \\
\hline 23 & & $2 A Q 8$ & Oxidoreductase & -8.8 & ADDGGGIIIIIILKKMMFFPSSTV \\
\hline 24 & & 2NV6 & Oxidoreductase & -8.03 & AADDGGGIIIIIILKMMFFPSTWYV \\
\hline
\end{tabular}


Citation: Mafakheri M and Sardari S (2016) Similarity in the Amino Acid Sequences of Mycobacterium tuberculosis Protein Targets Involved in Binding Sites of Docking with Thiacetazone. Pharm Anal Acta 7: 509. doi:10.4172/2153-2435.1000509

Page 3 of 7

\begin{tabular}{|c|c|c|c|c|c|}
\hline 25 & & $2 \mathrm{H} 9 \mathrm{I}$ & Oxidoreductase & -7.98 & ADDGGGIIIIIILLKMMMFFPSSTWTV \\
\hline 26 & & 3OEY & Oxidoreductase & -7.91 & ADDGGGIIIIIILKMMFFPSSTV \\
\hline 27 & & 2B37 & Oxidoreductase & -7.89 & ADDGGGIIIIILKMMFFPSSTTV \\
\hline 28 & \multirow{11}{*}{ B } & $1 \mathrm{P} 45$ & Oxidoreductase & -7.77 & AADDGGGIIIIIILKMMFFPSSTV \\
\hline 29 & & 1ENZ & Oxidoreductase & -7.76 & AADDGGGIIIIILKMFFFPSTV \\
\hline 30 & & 3OEW & Oxidoreductase & -7.72 & ADDGGGIIIIIILKMFFPSSTV \\
\hline 31 & & $2 \mathrm{AQK}$ & Oxidoreductase & -7.64 & AADDGGGIIIIIILKMFFPSTV \\
\hline 32 & & 3OF2 & Oxidoreductase & -7.58 & ADDGGGIIIIIILKMFFPSSTV \\
\hline 33 & & $2 \mathrm{AQH}$ & Oxidoreductase & -7.57 & ADDGGGIIIIILKMFFPSSTVV \\
\hline 34 & & 1 ENY & Oxidoreductase & -7.57 & ADDGGGIIIIIILKMFFPSSTV \\
\hline 35 & & $2 \mathrm{H} 7 \mathrm{P}$ & Oxidoreductase & -7.32 & ADDGGGIIIIIILKMFFPSSTV \\
\hline 36 & & $2 \times 22$ & Oxidoreductase & -7.27 & AADDGGGIIIIIILLKMMFFPSSTV \\
\hline 37 & & $2 \mathrm{H} 7 \mathrm{~L}$ & Oxidoreductase & -7.25 & ADDGGGIIIIIILKMMFFPSSTV \\
\hline 38 & & $2 \mathrm{H} 7 \mathrm{M}$ & Oxidoreductase & -6.97 & ADDGGGIIIIIILMMFFPSSTV \\
\hline 39 & \multirow{2}{*}{ C1 } & $3 F 69$ & Transferase & -7.93 & AANDEGGLLKMFTW \\
\hline 40 & & $3 F 61$ & Transferase & -7.85 & AANDEGGLKKMMMFSTVVV \\
\hline 41 & \multirow{2}{*}{$\mathrm{C} 2$} & 1DF7 & Oxidoreductase & -7.5 & AARRRDQQGGGGGGGIIILLSTWYV \\
\hline 42 & & 1DG5 & Oxidoreductase & -7.4 & AARRRRDQQGGGGGGGIIILLSSTWYV \\
\hline 43 & \multirow{2}{*}{$\mathrm{C} 3$} & 3HEM & \multirow{2}{*}{ Transferase } & -8.4 & CEGGHILLFFYYYY \\
\hline 44 & & $1 \mathrm{KPI}$ & & -8.39 & CEGGIILLLFFTWYYYY \\
\hline 45 & \multirow{2}{*}{$\mathrm{C} 4$} & 3HA5 & \multirow{2}{*}{ Transferase } & -7.85 & AQEEGGGHIILLFSTTWY \\
\hline 46 & & 2FK8 & & -7.69 & ACQQEEGGGHIILLFSSTTWY \\
\hline 47 & \multirow{2}{*}{ C5 } & 2WGE & \multirow{2}{*}{ Transferase } & -7.84 & ACGGHHFFFPTTV \\
\hline 48 & & $2 A Q B$ & & -7.41 & ADCGGHHMFFFPTTV \\
\hline 49 & \multirow{2}{*}{$\mathrm{C} 6$} & 1L1E & \multirow{2}{*}{ Transferase } & -7.23 & ARCQEEGGGGHILLFSSTTFT \\
\hline 50 & & $3 \mathrm{HA} 3$ & & -7.21 & AQEEGGGHIILFSTTWY \\
\hline 51 & \multirow{2}{*}{$\mathrm{C} 7$} & 2Q1Y & & -7.04 & AAARNNEEGGGGGGGGGLFPTT \\
\hline 52 & & $1 \mathrm{RLU}$ & Cell Cycle Signaling Protein & -6.93 & AAARNNDEEGGGGGGGGGLFPTT \\
\hline 53 & \multirow{3}{*}{$\mathrm{C} 8$} & 1RQ7 & & -6.93 & ARNNDEEGGGGGGGLFPT \\
\hline 54 & & $1 \mathrm{QPN}$ & \multirow{2}{*}{ Transferase } & -6.96 & AARRDGGHHLKST \\
\hline 55 & & $1 \mathrm{QPQ}$ & & -6.65 & RRHLLKKST \\
\hline 56 & \multirow{5}{*}{ C9 } & 3PYF & \multirow{2}{*}{ Transferase } & -6.72 & AANDDGGGGLLLMPV \\
\hline 57 & & 3PTY & & -5.47 & AANDDGGGGLLLMPV \\
\hline 58 & & $2 A 8 X$ & Oxidoreductase & -8.83 & $\begin{array}{l}\text { AAADCCNEGGGGGGGGGHILLKFFPPTYYY } \\
\text { VVV }\end{array}$ \\
\hline 59 & & $1 \mathrm{KPG}$ & Transferase & -8.39 & AACQQGGGGHILLFSSTTWYYV \\
\hline 60 & & $1 \mathrm{M} 4 \mathrm{I}$ & Transferase & -7.94 & ADDDDDEGFFSSTW \\
\hline
\end{tabular}


Citation: Mafakheri M and Sardari S (2016) Similarity in the Amino Acid Sequences of Mycobacterium tuberculosis Protein Targets Involved in

\begin{tabular}{|c|c|c|c|c|c|}
\hline 61 & & $1 \times 8 \mathrm{~A}$ & Theoretical Models & -7.77 & ADCHIFTYYYV \\
\hline 62 & & 1EYE & Transferase & -7.75 & RNNDDDGLKMFSVVVH \\
\hline 63 & & $2 \mathrm{HW} 2$ & Transferase & -7.72 & ANGGGLLLLKKMFFSTWVV \\
\hline 64 & \multirow{7}{*}{ M } & 2WGG & Transferase & -7.56 & AAAEGILPV \\
\hline 65 & & 1N4G & Oxidoreductase & -7.44 & AFTWVV \\
\hline 66 & & 3PYE & Transferase & -7.2 & DGHLSTYY \\
\hline 67 & & 2WGF & Transferase & -6.629 & ELLV \\
\hline 68 & & $3 G W C$ & Transferase & -6.24 & RRRHHHHM \\
\hline 69 & & $30 \times \mathrm{H}$ & Hydrolase inhibitor & -6.73 & ANHHIMY \\
\hline 70 & & 1NKT & Protein Transport & -8.57 & RRNDDQQEGGGLFPTTW \\
\hline
\end{tabular}

Table 1: Mycobacterium targets that docked with thiacetazone.

\section{Results}

There were some repeated patterns in amino acid sequences involved in binding site of thiacetazone and protein targets, among those targets. It was found out category A, B and C. Category A and B belong to the transferase and oxidoreductase classes, respectively. Category $\mathrm{C}$ consist of various target's classes such as oxidoreductase, transferase and the cell cycle signaling protein classes (Table 1). Figure 2 represents the frequency of amino acids involved in binding sites of our 70 Mycobacterium protein targets and thiacetazone.

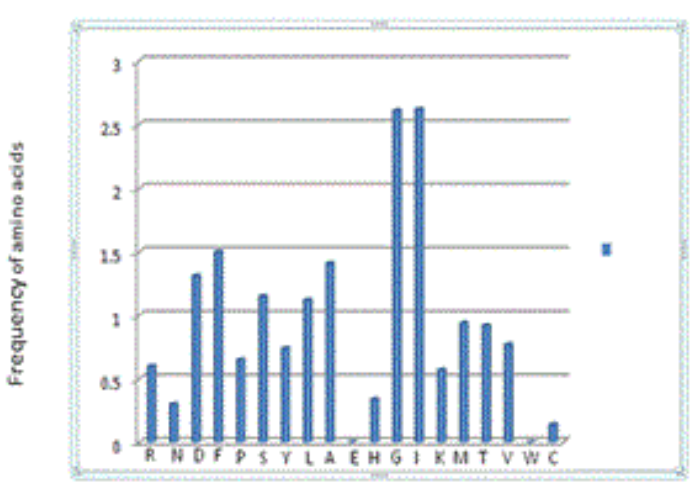

Amino acid name

Figure 2: Frequency of amino acids in binding sites of Mycobacterium protein targets and thiacetazone.

\section{Category A}

This category consists of 8 targets: 1GSI, 1MRN, 1G3U, 1W2G, 1N5J, 1MRS, 1N5I, 1W2H (Figure 3). All of them belong to transferase class and form about $11 \%$ of these Mycobacterium targets. Free energy of binding in this Category is the least $(-8.59-9.55 \mathrm{kcal} / \mathrm{mol})$. Amino acids sequences of their binding sites and the rate of similarity represent in Table 1.
Figure 4 shows a diagram of frequency belong to amino acids involved in binding sites of thiacetazone- target in group A. Table 1 also shows some diversity, in the case of $1 \mathrm{MRN}$ and though $1 \mathrm{~W} 2 \mathrm{G}$, $1 \mathrm{~N} 5$ J, 1MRS, $1 \mathrm{~N} 5 \mathrm{I}$ and $1 \mathrm{~W} 2 \mathrm{H}$ have the same sequences but there is also a bit different. Generally, except $1 \mathrm{MRN}$ their binding sites begin with 2 arginine molecules and end with 2 to 4 tyrosine molecules. The number of amino acids in binding site of this target are 21 whereas others have only 10-12 (Figure 3 ).

\begin{tabular}{|c|c|c|}
\hline $1 \mathrm{GSI}$ & --RRNDE - - - - - FFPSYYY - & 12 \\
\hline $1 \mathrm{~W} 2 \mathrm{H}$ & --RRNDL - - - - - FFPSYY - - & 11 \\
\hline $1 \mathrm{MRN}$ & ARRRNDDDEEEHLFFPSYYYY & 21 \\
\hline $1 \mathrm{~N} 5 \mathrm{I}$ & -RRNDD - . . - - LFPSYYY - & 12 \\
\hline $1 \mathrm{GSI}$ & --RRND - . - . - FFPSYYY - & 11 \\
\hline $1 \mathrm{G} 3 \mathrm{U}$ & -RRND - - - - - FF PSYYY - & 11 \\
\hline 1MRS & --RRND - - - - - - L FPSYYY - & 11 \\
\hline \multirow[t]{2}{*}{$1 \mathrm{~W} 2 \mathrm{G}$} & --RRN - - - - F FPSYYY- & 10 \\
\hline & $: * * * * *$ & \\
\hline \multicolumn{3}{|c|}{$\begin{array}{l}\text { Figure 3: Multiple sequence alignments of amino acids in binding } \\
\text { site involved in docking between thiacetazone and protein targets } \\
\text { belong to category A. }\end{array}$} \\
\hline
\end{tabular}


Citation: Mafakheri M and Sardari S (2016) Similarity in the Amino Acid Sequences of Mycobacterium tuberculosis Protein Targets Involved in

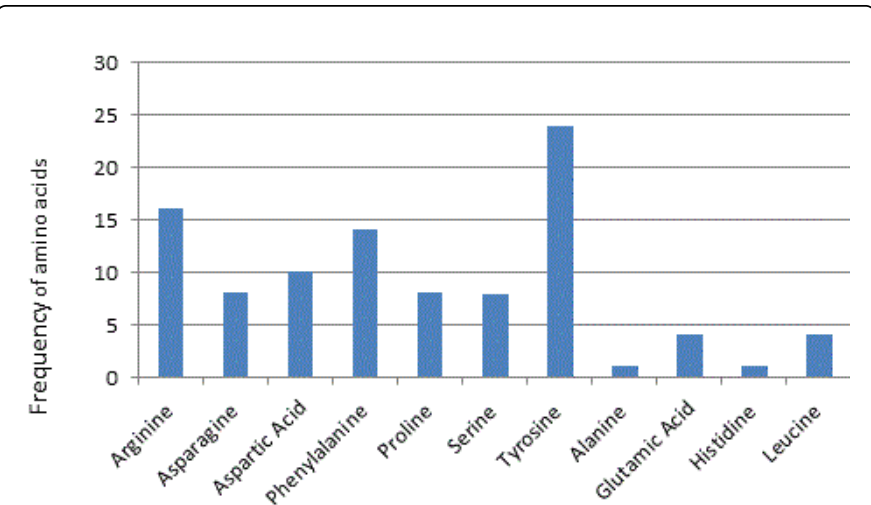

Figure 4: Frequency of amino acids involved in thiacetazone target binding site in group $\mathrm{A}$.

\section{Category B}

This category consist of 30 targets: 3FNF, 2PR2, 2IEB, 1ZID, 3FNH, 3FNG, 2 IDZ, 2 IED, 1p44, 2B35, 2B36, 2NSD, 2AQI, 2AQ8, 2H7N, 2NV6, 2H9I, 3OEY, 2B37, 1P45, 1ENZ, 3OEW, 2AQK, 3OF2, 2AQH, 1ENY, 2H7P, 2X22, 2H7L , 2H7M (Figure 5). This group with one hydrophobic amino acid, alanine beginning and another hydrophobic amino acid, valine in the end form about $43 \%$ of these Mycobacterium targets (Figure 5) $[15,16]$. All of them belong to the oxidoreductase class and free energy of binding in this category is between-8.91_-6.97 $\mathrm{kcal} / \mathrm{mol}$ (Table 1).

\section{Category C}

There are 9 small groups that consist of only two or three members and form about $27 \%$ of these targets. They are also very similar in Sequence of amino acids in binding site (Table 1).

The rest of 13 targets have no similarity in binding site amino acid sequences they are indicated as category M. Table 1 Mycobacterium targets that docked with thiacetazone.

Figure 6 shows a diagram of frequency belong to amino acids involved in binding sites of thiacetazone - target in group B.

\section{Discussion}

Results of multiple sequence alignment of protein targets belong to category A and B via ClustalW server represent a huge degree of similarity among the amino acids sequences involved in binding site in docking between thiacetazone and protein targets (Figures 2). Figure 3 and 4 represent frequency of amino acids involved in thiacetazone target binding site in group A and B respectively. We found out high frequency of arginine and tyrosine in group A binding sites which meets the results have represented in prior studies [23, 24]. Protein targets belong to category A have the least free energy of binding (-8.59 _ $-9.55 \mathrm{kcal} / \mathrm{mol}$ ) among these 70 protein targets.

\begin{tabular}{|c|c|c|}
\hline $1 \mathrm{P} 45$ & AADDGGG -IIIIIL - - KMMFFPSS- -T V- & 24 \\
\hline 1P44 & - ADDGGG -IIIIIL - - KMMFFPSS- -T V- & 23 \\
\hline $3 \mathrm{FNH}$ & AADDGGG IIIIL K -MMFFFPSS - -T V- & 26 \\
\hline $2 \mathrm{~B} 36$ & - ADDGG - - IIIIIL K - MMMFFPSS - -TV - & 23 \\
\hline $2 \mathrm{H} 9 \mathrm{I}$ & - ADDGGGIIIIIILL K - MMMFFPSSTWTV- & 27 \\
\hline 3 FNG & AADDGGG - IIIIL - - LKMFFPSS - -YV- & 24 \\
\hline $2 \mathrm{AQH}$ & - ADDGGG - IIIII - - - - LKMFFPSS- -T VV & 22 \\
\hline 2B37 & - ADDGGG - IIIIIL - - K MMFFPSS- -T TV & 24 \\
\hline $2 \mathrm{H} 7 \mathrm{M}$ & - ADDGGG - IIIIL - - - MMFFPSS- -TV - & 22 \\
\hline $3 O E Y$ & - ADDGGG - IIIIIL - - K MMFFPSS- -T V- & 23 \\
\hline $2 \mathrm{AQI}$ & - ADDGGG - IIIIIL - - K MMFFPSS- -T V- & 23 \\
\hline 2B 35 & - ADDGGG - IIIIII - - K MMFFPSS- -T V- & 23 \\
\hline 2NSD & - ADDGGG - IIIIIL - - K MMFFPSS- -T V- & 23 \\
\hline $2 \mathrm{H} 7 \mathrm{~N}$ & - ADDGGG - IIIIII - - K MMFFPSS- -T V- & 23 \\
\hline $2 \mathrm{H} 7 \mathrm{~L}$ & - ADDGGG - IIIIIL - - K MMFFPSS- -T V- & 23 \\
\hline
\end{tabular}

Figure 5: Multiple sequence alignment of amino acids sequences involved in binding site in docking between thiacetazone and protein targets belong to category $\mathrm{B}$.

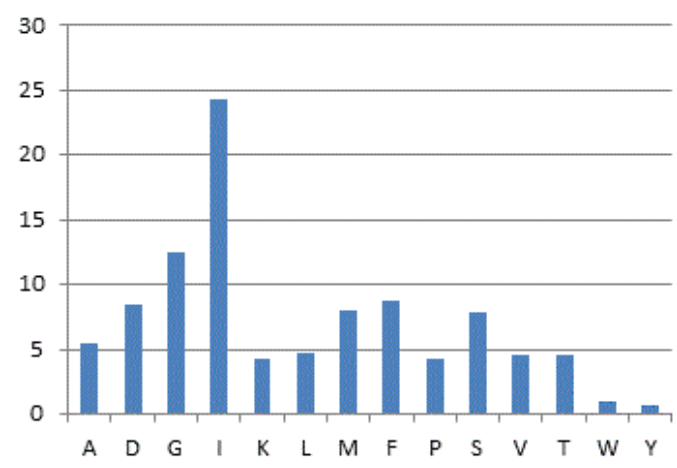

Figure 6: Frequency of amino acids involved in thiacetazonetarget's binding site in group $B$.

The amino acids sequences of their binding sites begin with 2 arginine molecules which on the basis of its geometry, charge distribution and its ability to form multiple $\mathrm{H}$-bonds are ideal for binding negatively charged groups such as thiacetazone with six potential negatively charged atoms (one oxygen, four nitrogen and one sulphur). On the other, hand these binding sites end with 2 to 4 tyrosine molecules which because their ability to make $\pi$ interactions and their hydrophobic surface area are generally most abundant 
residues in all binding sites and it is not surprisingly that the free energy of binding in this category is maximum [25-27] $1 \mathrm{MRN}$ begin with one alanine molecule which is second in rate of occurrence, accounting in a sample of 1150 protein [28] and then 3 arginine molecules. The arginine positive charge plus alanine hydrophobicity make it the second less free energy of binding in docking with in category A (Table 1). As Dennis A. Dougherty opinion that many drug-receptor interactions involve cation $-\pi$ interactions, ammonium group belongs to thiacetazone in one side and aromatic ring available in arginine or tyrosine in binding site of group A and B respectively on the other side may involve in drug-receptor interactions [29].

As Pearson mentioned significant similarity can be to be homologous [24] and as Gary D. Stormo declared homologous sequences usually have the same, or very similar, functions [26] although there are some evidences to promote this idea that imidazo [1,2-c] pyrimidin-4-ol derivatives as antitubercular agents. One of their compound showed the highest docking score and H-bond interaction with Arg140 and Gly19 that was also confirmed by single crystal X-ray analysis. The in silico results are also validated with in vitro antitubercular activity of compound $7 \mathrm{t}$. Compound $7 \mathrm{~b}$ exhibited in vitro antitubercular activity [30].

Surekha et al., also used ClustalW application to Sequence alignment, found out the amino acid residues (Met1, Asp2, Glu43, Ala44, Glu47, Lys51, Ala157 and Leu158). We also found frequently repeated amino acid sequences of $M$. tuberculosis protein targets involved in binding sites of docking with thiacetazone [31].

Pulaganti.et al., (2014) performed a systematic study was conducted to get an insight about Mtb-OSBS enzyme and the corresponding inhibitors using in silico methods. The active site amino acids have been identified by comparing the template sequence with the MtbOSBS sequence. They identified that Lys (108), Asn (140), Asp (138), Lys (110), Glu (189), Ser(236), Asp (188), Arg (27), Tyr (52), and Ser (237) are highly conserved, and these may play a vital role as active residues, similar to that in template protein.

Molecular modeling and docking studies of O-succinyl benzoate synthase of M. tuberculosis-a potential target for antituberculosis drug design [32]. Surekha.et al., (2016) in the study of OPRTase as an anti-pathogenic target, a homology model of OPRTase was constructed using $2 \mathrm{P} 1 \mathrm{Z}$ as a template. About $100 \mathrm{~ns}$ molecular dynamics simulation was performed to investigate the conformational stability and dynamic patterns of the protein. The aminoacid residues (Met1, Asp2, Glu43, Ala44, Glu47, Lys51, Ala157 and Leu158) lining in the binding site were predicted using Site Map. The amino acid residues (Met1, Asp2, Glu43, Ala44, Glu47, Lys51, Ala157 and Leu158) lining in the binding site were predicted using Site Map, a study that may provide better insight for designing potent anti-pathogenic agent [31].

Investigation of vital pathogenic target orotate phosphoribosyl transferases (OPRTase) from Thermus thermophilus HB8: Phylogenetic and molecular modeling approach [28].

\section{Conclusion}

Although many countries in sub-Saharan Africa still use extremely cheap thiocetazone, but severe (sometimes fatal) skin reactions in HIV positive patients due toit, lead to decline its usage and promote researches for synthesis of its other analogues to find out an new alternative has been performed concomitantly. Awareness about targets, rate of their maximum free energy of binding and their sequence of amino acids in binding site might be necessary for designing the new drugs that meet primary criteria. The implications of this study may be important for the design of those analogues. Although there are some evidences to promote this idea that the case of "function" is more complicated as the same enzyme have "different" roles in two tissues because of different circumstance but this method can be a good route for predicting of binding strength. Such methods may be present a good route for prediction hence as Stormo [26] mentioned we have expected that new agent with similar amino acids sequences involved in binding site in docking with protein targets has the same, or very similar, functions. Admittedly the second but more important step should be finding the function of these similar sequences.

\section{References}

1. (2016) Tuberculosis, Media centre. World Health Organization.

2. (2010) Tuberculosis Fact sheet $\mathrm{N}^{\circ} 104 "$. World Health Organization.

3. Asghari M, Heidarzadeh S, VaiseMalekshahi Z, Hemmatzadeh M, Razzaghe, Karimi MJ, et al. (2012) The Epidemiology of Tuberculosis in Tabriz, Iran: A Five Year Retrospective Study. J Med Bacteriol 1: 23-30.

4. Metanat M, Salehi MB, Sharifi mood B, Jahantigh AR, Rouhani Z (2006) Epidemiology of extra pulmonary tuberculosis in Zahedan. Tabib-EShargh 7: 275-281.

5. (2016) Tuberculosis (TB) Multidrug-resistant tuberculosis (MDR-TB), World Health Organization (WHO).

6. (2012) Tuberculosis (TB) Frequently asked questions - XDR-TB, World Health Organization (WHO).

7. Migliori GB, De Iaco G, Besozzi G, Centis R, Cirillo DM (2007) First tuberculosis cases in Italy resistant to all tested drugs. Euro Surveill 12.

8. Parida SK, Axelsson-Robertson R, Rao MV, Singh N, Master I, et al. (2015) Totally drug-resistant tuberculosis and adjunct therapies (Review). J Intern Med 277: 388-405.

9. Shokouhi S, AlaviDarazam I (2015) Drug-Resistant Tuberculosis and Group 5 Anti-Tuberculosis Drugs. Archives of Pediatric Infectious Diseases 3.

10. Velayati AA, Masjedi MR, Farnia P, Tabarsi P, Ghanavi J, et al. (2009) Emergence of new forms of totally drug-resistant tuberculosis bacilli: super extensively drug-resistant tuberculosis or totally drug-resistant strains in iran. Chest 136: 420-425.

11. (2016) What is multidrug-resistant tuberculosis (MDR-TB) and how do we control it. World Health Organization.

12. (2014) FDA Approval of Bedaquiline - The Benefit-Risk Balance for Drug-Resistant Tuberculosis. N Engl J Med 371: 689-691.

13. Dooley KE, Obuku EA, Durakovic N, Belitsky V, Mitnick C, et al. (2013) World Health Organization Group 5 Drugs for the Treatment of DrugResistant Tuberculosis:Unclear Efficacy or Untapped Potential? J Infect Dis 207: 1352-1358.

14. Nunn P, Brindle R, Wasunna K, Brindle R, Imalingat A, et al. (1991) Cutaneous hypersensitivity reactions due to thiacetazone in HIV-1 seropositive patients treated for tuberculosis. Lancet 337: 627-630.

15. Sahi SP, Chandra K (1974) Thiacetazone induced Steven Johnson syndrome: a case report. Indian J Chest Dis 16: 124-125.

16. Coxon GD, Craig D, Corrales RM, Vialla E, Gannoun-Zaki L, et al. (2013) Synthesis, antitubercular activity and mechanism of resistance of highly effective thiacetazoneanalogues. PLoS One 8.

17. Esfahanizadeh M, Omidi K, Kauffman J, Gudarzi A, Shahraki Zahedani S, et al. (2014) Synthesis and evaluation of new fluorinated anti-tubercular compounds. Iran J Pharm Res 13: 115-126.

18. Kandasamy S, Hassan S, Gopalaswamy R, Narayanan S (2014) Homology modelling, docking, pharmacophore and site directed mutagenesis analysis to identify the critical amino acid residue of PknI from $M$. tuberculosis. J Mol Graph Model 52: 11-19. 
Citation: Mafakheri M and Sardari S (2016) Similarity in the Amino Acid Sequences of Mycobacterium tuberculosis Protein Targets Involved in Binding Sites of Docking with Thiacetazone. Pharm Anal Acta 7: 509. doi:10.4172/2153-2435.1000509

Page 7 of 7

19. Pulaganti M, Banaganapalli B, Mulakayala C, Chitta SK, CMA (2014) Molecular modeling and docking studies of $\mathrm{O}$-succinylbenzoate synthase of M. tuberculosis--a potential target for antituberculosis drug design. Appl Biochem Biotechnol 172: 1407-1432.

20. Surekha K, Prabhu D, Richard M, Nachiappan M, Biswal J, et al. (2016) Investigation of vital pathogenic target orotatephosphoribosyltransferases (OPRTase) from Thermusthermophilus HB8: Phylogenetic and molecular modeling approach. Gene 583: 102-111.

21. A Structural View of Biology, An Information Portal to 124286 Biological Macromolecular Structures.

22. http://www.arguslab.com

23. http://www.ebi.ac.uk/Tools/msa/ClustalW2/

24. Anslyn EV, Dougherty D (2006) Modern Physical Organic Chemistry.

25. Villar HO, Kauvar LM (1994) Amino acid preferences at protein binding sites. FEBS Lett 349: 125-130.
26. Pearson WR (2014) An Introduction to Sequence Similarity ("Homology") Searching. Curr Protoc Bioinformatics.

27. Stormo GD (2009) An Introduction to Sequence Similarity ("Homology"). Current Protocols in Bioinformatics.

28. Kumar S, Kumar N, Kumar Gaur R (2011) Amino Acid Frequency Distribution at Enzymatic Active Site.

29. The IIOAB J [serial online] 10th-May- 2011.[ cited 2015 Apr 10] Vol. 2; Issue 4; 2011: 23-30.

30. Buki Kwon, Palinda Ruvan Munashingha, Yong-Keol Shin, Chul-Hwan Lee (2016) Physical and functional interactions between nucleosomes and Rad27, a critical component of DNA processing during DNA metabolism. The FEBS J.

31. Doolittle RF (1989) Prediction of Protein Structures and the Principles of Protein Conformation, New York, Plenum, pp: 599-623.

32. Dougherty D (2013) The cation- $\pi$ interaction. Acc Chem Res 46: 885-893. 